\title{
Reconstruction of Surgical Defect in Mandible Using Patient Specific Implant - A Case Report
}

\section{Shweta Murlidhar Patil*, Nitin Bhola, Chinmay Ghavat and Anchal Agarwal}

Department of Oral and Maxillofacial Surgery, Sharad Pawar Dental College and Hospital, India

*Corresponding Author: Shweta Murlidhar Patil, Department of Oral and Maxillofacial Surgery, Sharad Pawar Dental College and Hospital, India.
Received: May 24, 2021

Published: June 11, 2021

(C) All rights are reserved by Shweta

Murlidhar Patil., et al.

\section{Abstract}

The odontogenic keratocyst (OKC) is a commonly occurring developmental cystic lesion of the jaw with a predilection for males (1.3:1) in the $2^{\text {nd }}-3^{\text {rd }}$ decade [1]. It accounts to approximately $11 \%$ of all cysts of jaw and occurs most commonly in the posterior body or ramus of the mandible [2]. It's destructive nature and propensity for recurrence necessitates aggressive treatment to ensure low recurrence rate [3]. Autogenous bone has been a gold standard for reconstruction. Replacement of mandibular bone has been performed by using free fibula, scapula, iliac crest grafts etc. It is associated with donor site morbidity and difficulty to shape the autogenous bone in areas with curvature. Patient Specific Implants has revolutionised the reconstruction ladder and is a treatment modality for these arduous cases. In the present case of OKC a segmental resection with disarticulation was performed. PEEK (Polyether Etherketone) implant was used for reconstruction. PEEK has properties similar to bone and it overcomes disadvantages of titanium. Keywords: Odontogenic Keratocyst; Mandibular Reconstruction; Patient Specific Implant; DICOM; PEEK

\section{Introduction}

Ever since its first description by Philipsen in 1956, Odontogenic Keratocyst (OKC) continues to perplex surgeons with its unpredictable nature [4]. The most characteristic feature of OKC is its ability to recur at an exceptionally high rate owing to the satellite cells or daughter cells [5]. This has lead to surgeons advocating aggressive treatment modalities for OKC when compared to other odontogenic cysts. According to Morgan., et al. conservative treatment of the lesion includes enucleation (with or without curettage) or marsupialisation. The aggressive treatment comprises of peripheral ostectomy, chemical curettage with Carnoy's solution or en bloc resection. Large extensive lesions within the bone cause local destruction and require radical surgical approaches such as segmental resections followed by reconstruction to mini- mize the recurrence. Hence, the need for reconstructive treatment modalities arises. Reconstruction plates have been used considerable complication like plate breakage, loosening, infection. Nonvascularized autogenous bone grafts harvested from the ilium or microvascular composite tissue transfer from the fibula or the iliac crest have been used as immediate reconstructive modalities for reconstruction of the defect which hold a risk of infection, bone resorption, fragmentation and donor site morbidity [6]. As research continues, new treatment modality for reconstruction of such large defects is patient specific implant using PEEK. Polyether etherketone is an alloplastic biocompatible material which is used for $3 \mathrm{D}$ reconstruction of the surgical defect [8]. In the present case we have reconstructed the surgical defect post resection using a customized PEEK implant. 


\section{Case Report}

A 27 year old male reported to Oral Surgery OPD, Sharad Pawar Dental College and Hospital, Sawangi, Wardha, with a chief complain of a painful swelling over left side of face since 3 months. The swelling was small initially and gradually increased to the size of 7 x $4 \mathrm{cms}$ approximately. He experienced pain and gave history of restricted jaw movements. On palpation, the inspectory findings were confirmed and the lesion was found to be firm and bony hard in consistency and tender in nature. Egg shell crackling was evident in virtue of the buccal cortex expansion. A single left submandibular lymph node was palpable which was $2 \times 1 \mathrm{~cm}$ in size approximately, oval in shape, soft to firm in consistency and tender in nature.

CT Head was done which was suggestive of a well-defined expansile lytic lesion with well corticated borders measuring $5.5 \times 3.2$ x $2.9 \mathrm{cms}$ in the body, angle and ramus of the mandible on the left side involving the ipsilateral retromolar trigone, masseter muscle, medial and lateral pterygoid muscle. The lesion was unilocular cystic with air foci within and no solid component. An incisional biopsy was performed under local anesthesia and the histopathological diagnosis of Orthokeratinized Odontogenic Keratocyst (OKC) was made (Biopsy Number: 434/20).

Considering the extent of the lesion, perforation and thinning of the bone cortex we opted for resection of the cystic lesion of mandible and reconstruction of the defect formed was planned to be reconstructed using the customized PEEK implant. The customized PEEK implant was designed and fabricated using DICOM (Digital Imaging and Communications in Medicine) which was sent to the manufacturer, where $3 \mathrm{D}$ images using CAD/CAM software were created. CT Scan DICOM images were sent to the implant laboratory. An stereolithographic model was prepared. The PEEK implant was thereby fabricated and sterilised prior to its use.

Under general anesthesia, low Aprons incision was given and the expansile bony lesion was exposed. Resection margins were placed from 35 to the Head of condyle of left side and subsequent disarticulation was carried out. Resection was carried out as planned with the help of cutting guides. Reconstruction of the surgical defect was carried out by using the PEEK implant and titanium implant plates with screws, one at the superior and the other at the inferior border were used for fixation. The resected speci- men was sent for histopathological analysis and examination and the report was suggestive of Odontogenic Keratocystic lesion.

Post operatively patient was advised soft diet. The patient is on a follow up of 12 months, with no specific complaint or complications. No recurrence has been noted so far.
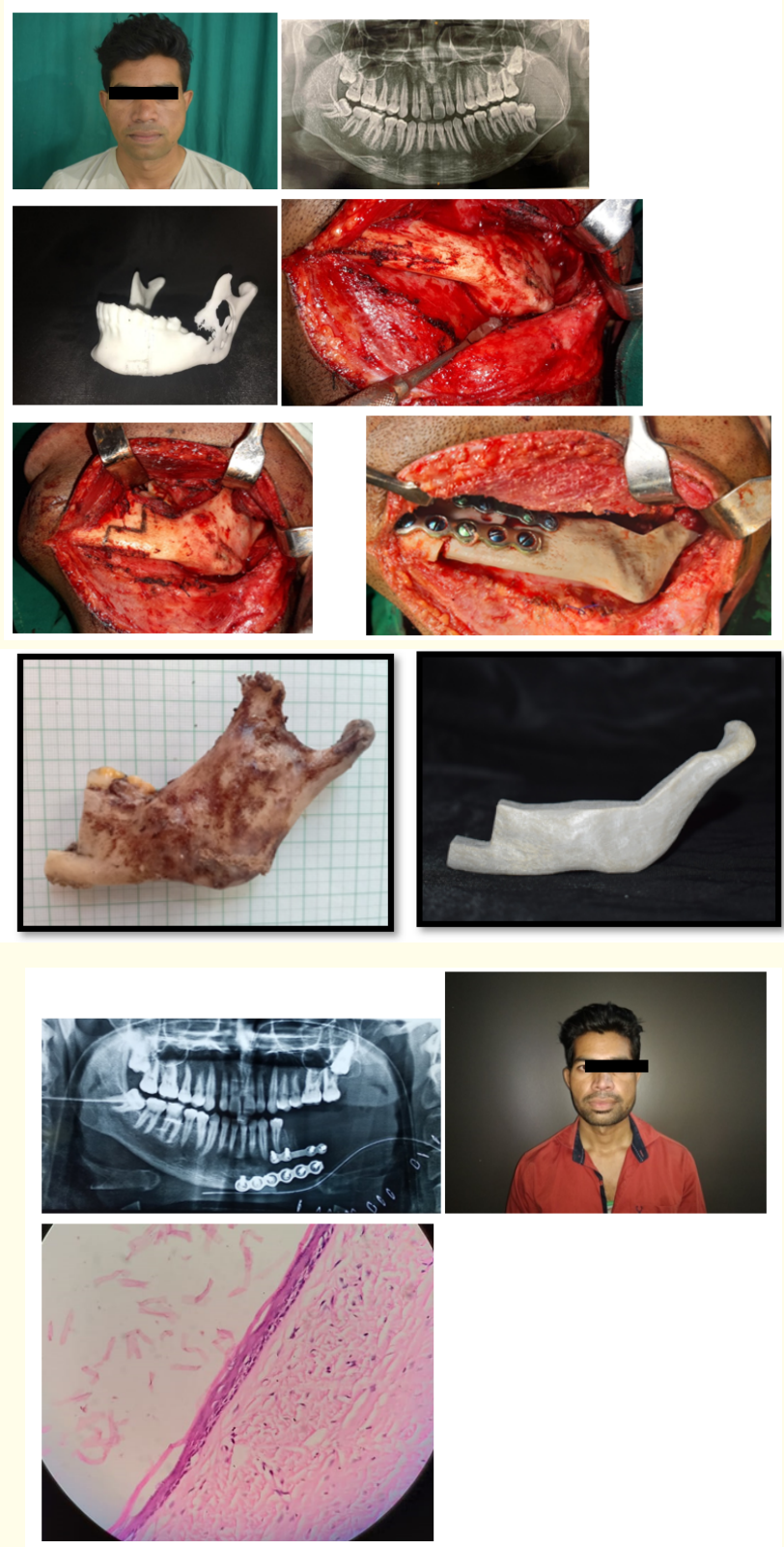

Figure 


\section{Discussion}

A cyst is a pathologic cavity having fluid, semifluid, or gaseous contents that are not created by the accumulation of pus, frequently, but not always, is lined by epithelium (Kramer, 1974). OKCs are common developmental odontogenic cysts and encompasses around $10-12 \%$ of all jaw cysts. OKCs usually occur in the second and third decades of life and possessing slight male predominance (1.3:1). Most of the OKCs occur in the body of the mandible in the molar region and vertical ramus region [9]. OKC generally occurs as a singular lesion. The etiology of this cysts originates from the dental lamina remnants in the maxilla and mandible. The patients who are symptomatic give symptoms of pain, swelling, expansion, drainage and bone perforation. Radiographically, it appears as a well-defined radiolucency and is mostly multilocular. The borders often display well defined corticated borders with a thin sclerotic border of bone [10]. OKC leads to displacement of teeth adjacent to the lesion. Very few shows root resorption [11]. Microscopically, the epithelial lining displays a characteristic 6 - 10 cell layer thick epithelium which shows palisading of basal cells and a thin parakeratinized lining layer giving it a corrugated appearance [9]. Consequent to the budding of the basal layer, "daughter cyst" formation is a frequent finding [5]. In contradiction with other odontogenic cysts, OKCs have a high recurrence rate, reportedly ranging from $25 \%$ to $60 \%$ [2]. Incomplete removal of the tenacious cystic lining is the most accepted theory for these high rates of recurrence [3].

The treatment modalities that have been recommended in the literature comprise of Enucleation, Enucleation with chemical fixation, Marsupialisation, Marsupialisation followed by enucleation, Resection or Radiotherapy (Cook, 1973). As indicated by Bramley (1970) the choice of treatment modality is largely dictated by the size, extent and location of the lesion. Occasionally, this may be achieved by supraperiosteal, rather than subperiosteal dissection without sacrificing the overlying mucosa. In the current case, given the lytic nature of the lesion and its extension, we opted for a segmental resection.

Often, as the disease affects individuals in their young age, reconstruction options with their various shortcomings, often pose an enigmatic question to the surgeon. Free tissue transfer (FTT), autografts, allografts, xenograft, reconstruction plates, metallic or non-metallic material alloplastic bone substitutes and Patient Specific Implants have been proposed as an option for the reconstruc- tion of discontinuity defects. The Reconstruction plate provides rigidity and stable occlusion. Contouring into desired shape is possible thereby eliminating the need for maxillomandibular fixation. It is economical and readily available. The possible complications are exposure of plate, loosening of screw, fracture of plate and screw, TMJ pain with trismus, skin contracture and tumour recurrence or metastasis [12]. The free fibula graft serves the greatest advantage of the ability to provide a large bone length even after total jaw resection. It is easier to harvest and provides rich periosteal blood supply. It provides adequate amount of bone width and height for functional reconstruction with osseointegrated implants and overdentures. It has low donor site morbidity and has a comparatively thicker cortex than radius, scapula and ilium. It may cause gait abnormalities due to chronic pain and discomfort. Bone resorption and partial necrosis of skin island when skin is used to reconstruct external defect is a huge disadvantage. As with any free flap there is considerable increase in the hospital stay. It also leads to delay in recovering active limb functions like walking and running.

The Non-vascularized graft provides adequate tension-free soft tissue, ease of placement and contouring of bone graft along with shaping of mandible and muscle insertion. It has a shorter rehabilitation duration. These grafts are restricted to only smaller defects (less than $6 \mathrm{~cm}$ ). The potential complications include infection, suture dehiscence, and subsequent loss of graft $[6,15]$.

Alloplastic implants have overcome the main disadvantage of bone resorption owing to the use of autogenous bone grafts. Patient specific implants (PSI) came into existence with an intention to acquire a customized implant fit. It bears the advantages of being faster along with greater accuracy with the additional benefit of shorter rehabilitation and general reduction in costs. PSI necessitates greater preoperative planning, preoperative CT scan or MRI, valuable information from the surgeon ${ }^{[16]}$. In unilateral defects the unaffected side may be used as a guide and computationally camouflaged on the affected side using specific "mirroring" software [17]. This grants for the design of a PSI that has the capacity for precise restoration of facial symmetry [18].

PEEK, is a semicrystalline thermoplastic polymer characterized by excellent mechanical and chemical properties, as well as by biologic safety, which makes this material a reliable alternative to the other alloplastic bone substitutes. It has beneficial biological and 
mechanical characteristics such that it is highly comparable to titanium and yet resembles the native bone. However, as opposed to titanium, PEEK has the ability to avoid thermal conduction that may have a detrimental effect on the brain but lacks the bioactivity for bony fusion. Computer-designed patient-specific PEEK implants for craniofacial reconstructive procedures are a feasible alternatives when autologous bone grafts are unavailable or not suited. Such pre-fabrication offers the leeway of reduced operative times through minimal to no intraoperative adjustments and the omission of additional surgical site. The fact that it can be coupled with CAD/CAM techniques, allowing the manufacture of custom-made implants, which can be finely tailored based on the individual's anatomy. The replaced surgical defect enables the restoration of function and aids in the establishment of esthetics [19,20].

The patient is on a 12 month follow up period and no complications regarding the surgery or the implant have been reported. There are no signs of the recurrence of the disease.

\section{Conclusion}

PEEK (Polyether ether ketone) is biocompatible, resistant to thermal and ionizing radiation and resembles cortical bone biomechanically. It is a radiolucent material and is capable of avoiding scattering of radiation if required. Another added advantage is it's light weight. These favourable characteristics have led to the increasing use of PEEK implants for craniofacial defects which remain a significant reconstructive challenge. Hence patient specific implant PEEK helps in restoration of the function with minimal disturbances of the aesthetics and prevents donor site morbidity.

\section{Bibliography}

1. Karandikar S., et al. "Large Odontogenic Keratocyst of Mandible".

2. Madras J and Lapointe H. "Keratocystic odontogenic tumour: reclassification of the odontogenic keratocyst from cyst to tumour". Journal of the Canadian Dental Association 74.2 (2008).

3. Anand VK., et al. "Malignant potential of the odontogenic keratocyst". Otolaryngology-Head and Neck Surgery 111.1 (1994): 124-129.

4. Toller PA. "Newer concepts of odontogenic cysts". International Journal of Oral Surgery 1.1 (1972): 3-16.
5. Myoung H., et al. "Odontogenic keratocyst: review of 256 cases for recurrence and clinicopathologic parameters". Oral Surgery, Oral Medicine, Oral Pathology, Oral Radiology, and Endodontology 91.3 (2001): 328-333.

6. Moura LB., et al. "Autogenous non-vascularized bone graft in segmental mandibular reconstruction: a systematic review". International Journal of Oral and Maxillofacial Surgery 45.11 (2016): 1388-1394.

7. Enucleation OA. Maxillofacial Surgery.

8. Manrique OJ., et al. "Craniofacial reconstruction using patientspecific implants polyether ether ketone with computer-assisted planning". Journal of Craniofacial Surgery 26.3 (2015): 663-666.

9. Blanchard SB. "Odontogenic keratocysts: review of the literature and report of a case". Journal of Periodontology 68.3 (1997): 306-311.

10. Brannon RB and Colonel L. "The odontogenic keratocyst: a clinicopathologic study of 312 cases, II: histologic features". Oral Surgery, Oral Medicine, Oral Pathology, and Oral Radiology 43 (1977): 233-255.

11. Koseoglu BG., et al. "Odontogenic cysts: a clinical study of 90 cases". Journal of Oral Science 46.4 (2004): 253-257.

12. Ueyama Y., et al. "Analysis of reconstruction of mandibular defects using single stainless steel AO reconstruction plates". Journal of Oral and Maxillofacial Surgery 54.7 (1996): 858862.

13. Guerra MF., et al. "Vascularized free fibular flap for mandibular reconstruction: a report of 26 cases". Journal of Oral and Maxillofacial Surgery 59.2 (2001): 140-144.

14. Peled M., et al. "The use of free fibular flap for functional mandibular reconstruction". Journal of Oral and Maxillofacial Surgery 63.2 (2005): 220-224.

15. Akbay E and Aydogan F. "Reconstruction of isolated mandibular bone defects with non-vascularized corticocancellous bone autograft and graft viability". Auris Nasus Larynx 41.1 (2014): 56-62. 
16. Baumann A., et al. "Late reconstruction of the orbit with patient-specific implants using computer-aided planning and navigation". Journal of Oral and Maxillofacial Surgery 73.12 (2015): S101-106.

17. Scolozzi P. "Applications of 3D orbital computer-assisted surgery (CAS)". Journal of Stomatology, Oral and Maxillofacial Surgery 118.4 (2017): 217-223.

18. Jonkergouw J., et al. "Outcome in patient-specific PEEK cranioplasty: a two-center cohort study of 40 implants". Journal of Cranio-Maxillofacial Surgery 44.9 (2016): 1266-1272.

19. Ghavat C., et al. "Reconstruction of mandibular defect using patient specific peek implant-An approach to aesthetic reconstruction". Medical Science 24.103 (2020): 1261-1266.

20. Alonso-Rodriguez E., et al. "Polyetheretherketone custommade implants for craniofacial defects: report of 14 cases and review of the literature". Journal of Cranio-Maxillofacial Surgery 43.7 (2015): 1232-1238.

Volume 5 Issue 7 July 2021

(c) All rights are reserved by Shweta Murlidhar Patil., et al. 